\title{
DEEP BROADBAND OBSERVATIONS OF THE DISTANT GAMMA-RAY BLAZAR PKS 1424+240
}

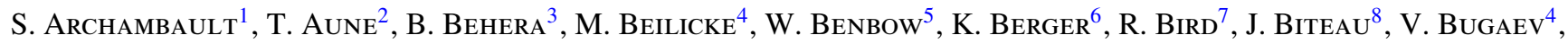

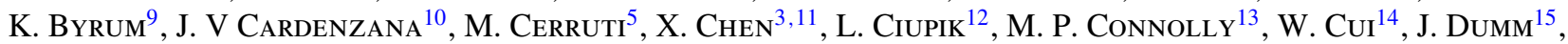
M. Errando ${ }^{16}$, A. Falcone ${ }^{17}$, S. Federici ${ }^{3,11}$, Q. Feng ${ }^{14}$, J. P. Finley ${ }^{14}$, H. Fleischiack ${ }^{3}$, L. Fortson ${ }^{15}$, A. Furniss ${ }^{18}$, N. Galante ${ }^{5}$, G. H. Gillanders ${ }^{13}$, S. Griffin ${ }^{1}$, S. T. Griffiths ${ }^{19}$, J. Grube ${ }^{12}$, G. Gyuk ${ }^{12}$, D. Hanna ${ }^{1}$, J. Holder ${ }^{6}$,

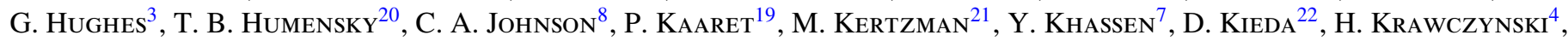
F. Krennrich ${ }^{10}$, S. Kumar ${ }^{6}$, M. J. Lang ${ }^{13}$, A. S Madhavan ${ }^{10}$, G. Maier ${ }^{3}$, A. McCanN ${ }^{23}$, K. Meagher ${ }^{24}$, P. Moriarty ${ }^{25}$, R. Mukherjee ${ }^{16}$, D. Nieto ${ }^{20}$, A. O'Faoláin de Bhróithe ${ }^{7}$, R. A. $\mathrm{ONG}^{2}$, A. N. Otte ${ }^{24}$, N. Park ${ }^{26}$, M. Pohl 3,11 , A. Popkow ${ }^{2}$,

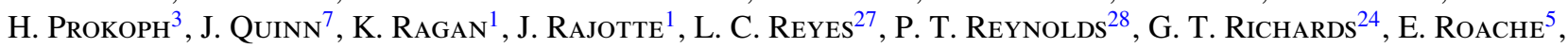
G. H. Sembroski ${ }^{14}$, K. Shahinyan ${ }^{15}$, D. Staszak ${ }^{1}$, I. Telezhinsky ${ }^{3,11}$, J. V. Tucci ${ }^{14}$, J. Tyler ${ }^{1}$, A. Varlotta ${ }^{14}$,

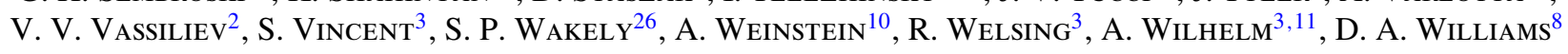
(The VERITAS COLlaboration)

M. AckermanN ${ }^{29}$, M. Ajello ${ }^{30}$, A. Albert ${ }^{31}$, L. Baldini ${ }^{32}$, D. Bastieri ${ }^{33,34}$, R. Bellazzini ${ }^{32}$, E. Bissaldi ${ }^{35}$, J. Bregeon ${ }^{36}$, R. Buehler ${ }^{29}$, S. Buson ${ }^{33,34}$, G. A. Caliandro ${ }^{31,37}$, R. A. Cameron ${ }^{31}$, P. A. Caraver ${ }^{38}$, E. Cavazzuti ${ }^{39}$, E. Charles ${ }^{31}$,

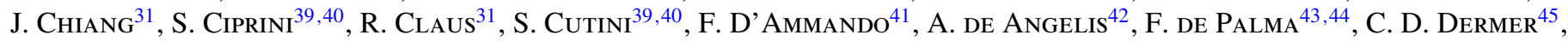
S. W. Digel ${ }^{31}$, L. Di Venere ${ }^{43}$, P. S. Drell ${ }^{31}$, C. Favuzzi ${ }^{43,44}$, A. Franckowiak ${ }^{31}$, P. Fusco ${ }^{43,44}$, F. Gargano ${ }^{44}$, D. Gasparrini ${ }^{39,40}$, N. Giglietto ${ }^{43,44}$, F. Giordano ${ }^{43,44}$, M. Girolettio ${ }^{41}$, I. A. Grenier ${ }^{46}$, S. Guiriec ${ }^{47,67}$, T. Jogler ${ }^{31}$,

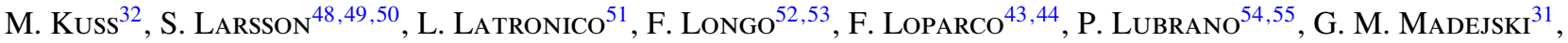
M. MaYer ${ }^{29}$, M. N. Mazziotta ${ }^{44}$, P. F. Michelson ${ }^{31}$, T. Mizuno ${ }^{56}$, M. E. Monzani ${ }^{31}$, A. Morselli ${ }^{57}$, S. Murgia ${ }^{58}$, E. Nuss $^{36}$, T. Ohsugi ${ }^{56}$, J. F. Ormes ${ }^{59}$, D. Paneque ${ }^{60}$, J. S. Perkins ${ }^{47}$, F. Piron ${ }^{36}$, G. Pivato ${ }^{34}$, S. Rainò ${ }^{43,44}$, M. Razzano ${ }^{32,68}$, A. Reimer ${ }^{31,61}$, O. Reimer ${ }^{31,61}$, S. Ritz ${ }^{62}$, M. SchaAl ${ }^{63,69}$, C. Sgrò ${ }^{32}$, E. J. Siskind ${ }^{64}$, P. SPinelli ${ }^{43,44}$, H. Takahashi ${ }^{65}$, L. Tibaldo ${ }^{31}$, M. Tinivella ${ }^{32}$, E. Troja ${ }^{47,66}$, G. Vianello ${ }^{31}$, M. Werner ${ }^{61}$, and M. Wood $^{31}$

(The Fermi LAT Collaboration)

${ }^{1}$ Physics Department, McGill University, Montreal, QC H3A 2T8, Canada

${ }^{2}$ Department of Physics and Astronomy, University of California, Los Angeles, CA 90095, USA

${ }^{3}$ DESY, Platanenallee 6, D-15738 Zeuthen, Germany

${ }^{4}$ Department of Physics, Washington University, St. Louis, MO 63130, USA

${ }^{5}$ Fred Lawrence Whipple Observatory, Harvard-Smithsonian Center for Astrophysics, Amado, AZ 85645, USA

${ }^{6}$ Department of Physics and Astronomy and the Bartol Research Institute, University of Delaware, Newark, DE 19716, USA

${ }^{7}$ School of Physics, University College Dublin, Belfield, Dublin 4, Ireland

${ }^{8}$ Santa Cruz Institute for Particle Physics and Department of Physics, University of California, Santa Cruz, CA 95064, USA

${ }_{9}^{9}$ Argonne National Laboratory, 9700 South Cass Avenue, Argonne, IL 60439, USA

${ }^{10}$ Department of Physics and Astronomy, Iowa State University, Ames, IA 50011, USA

${ }^{11}$ Institute of Physics and Astronomy, University of Potsdam, D-14476 Potsdam-Golm, Germany

12 Astronomy Department, Adler Planetarium and Astronomy Museum, Chicago, IL 60605, USA

${ }^{13}$ School of Physics, National University of Ireland Galway, University Road, Galway, Ireland

${ }^{14}$ Department of Physics, Purdue University, West Lafayette, IN 47907, USA

${ }^{15}$ School of Physics and Astronomy, University of Minnesota, Minneapolis, MN 55455, USA

${ }^{16}$ Department of Physics and Astronomy, Barnard College, Columbia University, NY 10027, USA

${ }^{17}$ Department of Astronomy and Astrophysics, 525 Davey Lab, Pennsylvania State University, University Park, PA 16802, USA

${ }^{18}$ Kavli Institute for Particle Astrophysics and Cosmology, SLAC National Accelerator Laboratory, Stanford University, Stanford, CA 94305, USA; amy.furniss@gmail.com

${ }^{19}$ Department of Physics and Astronomy, University of Iowa, Van Allen Hall, Iowa City, IA 52242, USA

${ }^{20}$ Physics Department, Columbia University, New York, NY 10027, USA

${ }^{21}$ Department of Physics and Astronomy, DePauw University, Greencastle, IN 46135-0037, USA

22 Department of Physics and Astronomy, University of Utah, Salt Lake City, UT 84112, USA

${ }^{23}$ Kavli Institute for Cosmological Physics, University of Chicago, Chicago, IL 60637, USA

${ }^{24}$ School of Physics and Center for Relativistic Astrophysics, Georgia Institute of Technology, 837 State Street NW, Atlanta, GA 30332-0430, USA

${ }^{25}$ Department of Life and Physical Sciences, Galway-Mayo Institute of Technology, Dublin Road, Galway, Ireland

${ }^{26}$ Enrico Fermi Institute, University of Chicago, Chicago, IL 60637, USA

${ }^{27}$ Physics Department, California Polytechnic State University, San Luis Obispo, CA 94307, USA

${ }^{28}$ Department of Applied Physics and Instrumentation, Cork Institute of Technology, Bishopstown, Cork, Ireland ${ }^{29}$ Deutsches Elektronen Synchrotron DESY, D-15738 Zeuthen, Germany

${ }^{30}$ Space Sciences Laboratory, 7 Gauss Way, University of California, Berkeley, CA 94720-7450, USA

${ }^{31}$ W. W. Hansen Experimental Physics Laboratory, Kavli Institute for Particle Astrophysics and Cosmology,

Department of Physics and SLAC National Accelerator Laboratory, Stanford University, Stanford, CA 94305, USA

${ }^{32}$ Istituto Nazionale di Fisica Nucleare, Sezione di Pisa, I-56127 Pisa, Italy

${ }^{33}$ Istituto Nazionale di Fisica Nucleare, Sezione di Padova, I-35131 Padova, Italy

${ }^{34}$ Dipartimento di Fisica e Astronomia “G. Galilei,” Università di Padova, I-35131 Padova, Italy

${ }^{35}$ Istituto Nazionale di Fisica Nucleare, Sezione di Trieste, and Università di Trieste, I-34127 Trieste, Italy

${ }^{36}$ Laboratoire Univers et Particules de Montpellier, Université Montpellier 2, CNRS/IN2P3, Montpellier, France

${ }^{37}$ Consorzio Interuniversitario per la Fisica Spaziale (CIFS), I-10133 Torino, Italy

${ }^{38}$ INAF-Istituto di Astrofisica Spaziale e Fisica Cosmica, I-20133 Milano, Italy

${ }^{39}$ Agenzia Spaziale Italiana (ASI) Science Data Center, I-00133 Roma, Italy

${ }^{40}$ Istituto Nazionale di Astrofisica - Osservatorio Astronomico di Roma, I-00040 Monte Porzio Catone (Roma), Italy

${ }^{41}$ INAF Istituto di Radioastronomia, I-40129 Bologna, Italy 


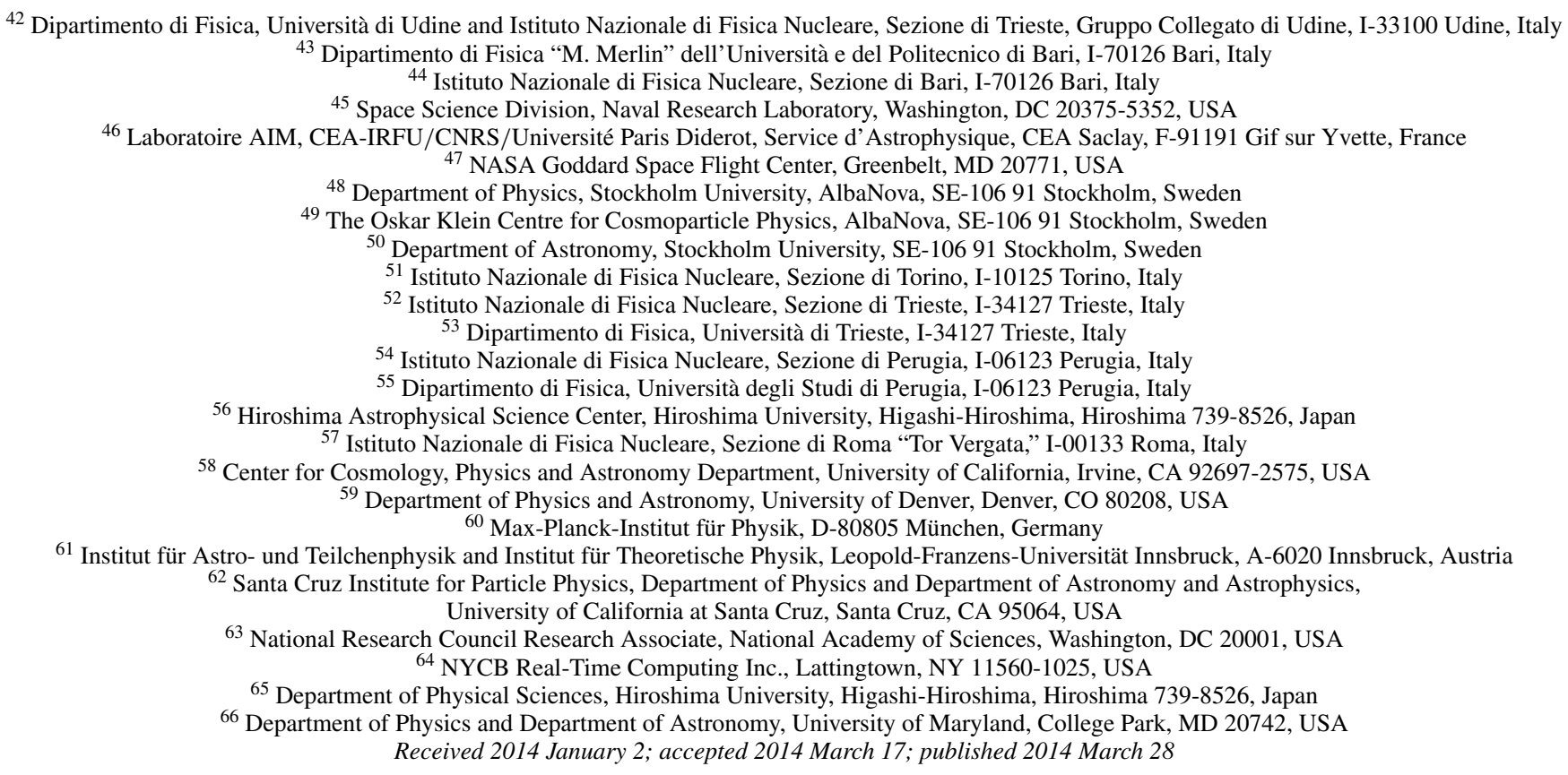

\section{ABSTRACT}

We present deep VERITAS observations of the blazar PKS 1424+240, along with contemporaneous Fermi Large Area Telescope, Swift X-ray Telescope, and Swift UV Optical Telescope data between 2009 February 19 and 2013 June 8 . This blazar resides at a redshift of $z \geqslant 0.6035$, displaying a significantly attenuated gamma-ray flux above $100 \mathrm{GeV}$ due to photon absorption via pair-production with the extragalactic background light. We present more than $100 \mathrm{hr}$ of VERITAS observations over three years, a multiwavelength light curve, and the contemporaneous spectral energy distributions. The source shows a higher flux of $(2.1 \pm 0.3) \times 10^{-7}$ photons $\mathrm{m}^{-2} \mathrm{~s}^{-1}$ above $120 \mathrm{GeV}$ in 2009 and 2011 as compared to the flux measured in 2013, corresponding to $(1.02 \pm 0.08) \times$ $10^{-7}$ photons $\mathrm{m}^{-2} \mathrm{~s}^{-1}$ above $120 \mathrm{GeV}$. The measured differential very high energy (VHE; $E \geqslant 100 \mathrm{GeV}$ ) spectral indices are $\Gamma=3.8 \pm 0.3,4.3 \pm 0.6$ and $4.5 \pm 0.2$ in 2009, 2011, and 2013, respectively. No significant spectral change across the observation epochs is detected. We find no evidence for variability at gamma-ray opacities of greater than $\tau=2$, where it is postulated that any variability would be small and occur on timescales longer than a year if hadronic cosmic-ray interactions with extragalactic photon fields provide a secondary VHE photon flux. The data cannot rule out such variability due to low statistics.

Key words: BL Lacertae objects: individual (PKS 1424+240)-cosmic background radiation gamma rays: galaxies

Online-only material: color figures

\section{INTRODUCTION}

PKS 1424+240 (VER J1427+237) is a distant very high energy (VHE; $E \geqslant 100 \mathrm{GeV}$ ) blazar at $z \geqslant 0.6035$ (Furniss et al. 2013). At this minimum distance, the intrinsic VHE emission is expected to be significantly absorbed by the extragalactic background light (EBL) via pair-production, $\gamma+\gamma \rightarrow e^{+}+e^{-}$ (Nikishov 1962). The absorption of VHE gamma rays by the EBL can be estimated using the model-dependent gamma-ray opacity, $\tau(E, z)$. The source flux, $F_{\text {int }}$, can be estimated from the observed flux, $F_{\text {obs }}$, using the relation $F_{\text {int }}=F_{\text {obs }} \times e^{\tau(E, z)}$.

The EBL cannot be directly measured due to foreground sources. The modification of distant VHE blazar spectra has been used to estimate the spectral properties of the EBL (Aharonian et al. 2006; Albert et al. 2008), providing photon

\footnotetext{
${ }^{67}$ NASA Postdoctoral Program Fellow, USA.

${ }^{68}$ Funded by contract FIRB-2012-RBFR12PM1F from the Italian Ministry of Education, University and Research (MIUR).

${ }^{69}$ Current address: Naval Research Laboratory, Washington, DC 20375, USA.
}

density upper limits consistent with the observational lower limits set by galaxy counts (Werner et al. 2004). Recent work has indicated that the EBL density is closer to the lower limits than the upper limits (Abramowski et al. 2013; Horns \& Meyer 2012; Ackermann et al. 2012). The distance to PKS 1424+240 makes the source ideal for studying extragalactic VHE photon propagation.

The high-energy spectral energy distribution (SED) measured in initial observations by VERITAS and the Fermi Large Area Telescope (LAT; Acciari et al. 2010) is investigated in Furniss et al. (2013), showing an absorption-corrected spectrum suggestive of VHE spectral hardening, though not beyond the conservative $\Gamma=1.5$ spectral limitation (where $d N / d E \propto$ $E^{-\Gamma}$ ) described in, e.g., Aharonian et al. (2006).

In an effort to understand the gamma-ray emission from PKS 1424+240, we analyze deeper observations by VERITAS and LAT, including more than four times the exposure in Acciari et al. (2010) and Furniss et al. (2013). In order to minimize hardening introduced from EBL absorption corrections, 
we explore the gamma-ray observations using the low-density "fixed" model from Gilmore et al. (2012). This model, which also provides compatible fits to LAT data in Ackermann et al. (2012), is comparable with that of Franceschini et al. (2008) used in Abramowski et al. (2013), and provides absorption corrections similar to other EBL models, e.g., Kneiske \& Dole (2010), Domínguez et al. (2011), and Finke et al. (2010). Luminosities calculated in this work use a $H_{0}=100 \mathrm{hm} \mathrm{s}^{-1} \mathrm{Mpc}^{-1}$, where $h=0.7$.

\section{OBSERVATIONS AND RESULTS}

\subsection{VERITAS}

VERITAS comprises four imaging atmospheric Cherenkov telescopes and is sensitive to gamma rays between $\sim 100 \mathrm{GeV}$ and $\sim 30 \mathrm{TeV}$ (Holder et al. 2006). The VERITAS observations of PKS 1424+240 were performed over three years. The first season (MJD 54881-55003) provides $28 \mathrm{hr}$ of quality-selected livetime and is reanalyzed here, showing results consistent with those reported in Acciari et al. (2010). The second season encompasses 14 quality-selected hours of observation between MJD 55598 and 55711, while the third season includes data spanning MJD 56334 to 56447, and provides $67 \mathrm{hr}$ of qualityselected livetime with a low threshold of $100 \mathrm{GeV}$, enabled by a camera upgrade in 2012.

The observations were taken at 0.5 offset in each of the four cardinal directions to enable simultaneous background estimation using the reflected-region method (Fomin et al. 1994). The recorded shower images are parameterized by their principal moments. Selection criteria are applied to the values of the mean scaled width (MSW), mean scaled length (MSL), apparent altitude of the maximum Cherenkov emission (shower maximum), and $\theta$, the angular distance between the position of PKS 1424+240 and the reconstructed origin of the event, giving an efficient suppression of the far more abundant cosmic-ray background. The cuts applied to all data are MSW $<1.1$, MSL $<1.3$, shower maximum $>7 \mathrm{~km}$, and $\theta<0$. 17 . These cuts were optimized a priori to yield the highest sensitivity for a soft $(\Gamma \sim 3.5)$ source with $5 \%$ of the Crab Nebula gammaray flux. ${ }^{70}$ These cuts are different from those in Acciari et al. (2010) because of improvements in the analysis software and detector simulation. The results are independently reproduced with two analysis packages (Cogan 2008; Prokoph 2013) and are summarized in Table 1. The same analysis was applied to data from the Crab Nebula for each season, providing compatible flux and spectral results with no evidence of an energy bias shift after the camera upgrade. In particular, the integrated fluxes measured above $200 \mathrm{GeV}$ agree to $11 \%$ or better $(1 \sigma$ confidence). The systematic uncertainty on the flux for a soft source like PKS $1424+240$ is estimated to be $\sim 40 \%$ and is regarded as constant for each of the observing periods.

The 2009 and 2011 observations show the source to have a flux of $4.6 \%$ of the Crab flux above $120 \mathrm{GeV}$, with indices of $\Gamma=3.8 \pm 0.3$ and $4.3 \pm 0.3$, respectively. The longer exposures obtained in 2009 and 2013 allow for the reconstruction of a significant spectral point in a higher energy bin than is possible with the 2011 data (see Figure 1). In an attempt to minimize a bias in the final spectral bin width, the energy binning is systematically determined, starting at $100 \mathrm{GeV}$, with bins of equal logarithmic width, initially corresponding to $15 \mathrm{GeV}$. The first bin that does not provide sufficient statistics for a

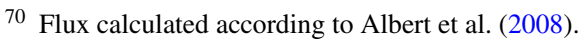

spectral point ( $<2$ standard deviations; $\sigma$ ), and is double in width compared to the preceding bin size. This wider bin is then utilized in the analysis to derive higher energy spectral points. The first instance where the doubling procedure does not provide a significant detection is reported with a $99 \%$ confidence level upper limit (Rolke et al. 2005), assuming the same spectral index that fit to the preceding bins. The spectral points are given at the energy corresponding to the event-weighted average in the bin. For the last bin, with bin edges $375 \mathrm{GeV}$ and $750 \mathrm{GeV}$, the weighted average corresponds to $510 \mathrm{GeV}$.

During the 2013 observations the source was in a dimmer VHE state of $2.2 \%$ Crab above $120 \mathrm{GeV}$ (see Figure 1). The VHE spectral index does not appear to change during this low state, displaying an index of $\Gamma=4.5 \pm 0.2$. The observations over each season are shown in the top panel of the light curve (Figure 2). The 2009 and 2013 observations show different states, with integral flux values above $120 \mathrm{GeV}$ of $(2.1 \pm 0.3) \times 10^{-7}$ photons $\mathrm{m}^{-2} \mathrm{~s}^{-1}$ and $(1.02 \pm 0.08) \times$ $10^{-7}$ photons $\mathrm{m}^{-2} \mathrm{~s}^{-1}$, respectively. Additionally, a constant fit to the VHE light curve shows less than a $1.1 \times 10^{-5}$ probability of a steady flux $\left(\chi^{2}=22.7\right.$ with 2 degrees of freedom; DOF). A search for variability above an opacity of $\tau=2$ (corresponding to $310 \mathrm{GeV}$ according to the Gilmore et al. $2012 \mathrm{EBL}$ model) does not provide significant evidence of variability given the very limited statistics at high energies, with integral flux values above $310 \mathrm{GeV}$ of $(5.6 \pm 3.8) \times 10^{-9} \mathrm{~m}^{-2} \mathrm{~s}^{-1}$ for $2009 / 2011$ combined data and $(3.6 \pm 1.8) \times 10^{-9} \mathrm{~m}^{-2} \mathrm{~s}^{-1}$ for 2013 data.

The power-law fit to the 2013 data is shown in Figure 3 with an envelope representing a $\pm 40 \%$ systematic error on the flux convolved with a systematic error on the index of \pm 0.3 . The data are corrected for absorption by the EBL assuming the model from Gilmore et al. (2012) at the minimum redshift of $z=0.6035$, resulting in a power-law fit $\left(\chi^{2} / \mathrm{DOF}=9.1 /\right.$ 9 , probability of 0.428 ) with index $\Gamma=3.0 \pm 0.4$. The 2009 absorption-corrected data provide a power-law fit with $\Gamma=2.8 \pm$ $0.7\left(\chi^{2} / \mathrm{DOF}=4.7 / 6\right.$, probability of 0.583$)$. As a consistency check, the data are also shown in Figure 3 with constant binning above $250 \mathrm{GeV}$. None of the individual points above $400 \mathrm{GeV}$ are statistically significant in this representation.

\subsection{Fermi LAT}

The Fermi LAT is a pair-conversion telescope sensitive to photons between $20 \mathrm{MeV}$ and several hundred GeV (Atwood et al. 2009). PKS 1424+240 is a bright gamma-ray source first reported in Abdo et al. (2009). Multiple epochs of LAT data are analyzed, including the complete Fermi LAT data set up to the time of analysis (MJD 54682 to 56452) and time intervals selected to be contemporaneous with the VERITAS observations, summarized in Table 1. The spectral parameters for the contemporaneous data are calculated using the unbinned maximumlikelihood method implemented in the LAT ScienceTools software package version v9r31p1, available from the Fermi Science Support Center. The spectral parameters for the full data set are calculated using the binned maximum-likelihood method. Only events from the "source" class with energy above $100 \mathrm{MeV}$ within a $12^{\circ}$ radius of PKS $1424+240$ with a zenith angle of $<100^{\circ}$ and detected when the spacecraft rocking angle was $<52^{\circ}$ are used. All sources within $12^{\circ}$ of the central source in the second LAT catalog (2FGL; Nolan et al. 2012) are included in the model. The normalizations of the components were allowed to vary freely during the spectral point fitting, which was performed using the instrument response function P7SOURCE_V6. The Galactic diffuse emission and an 
Table 1

Summary of Gamma-Ray Observations of PKS 1424+240

\begin{tabular}{|c|c|c|c|c|c|c|c|c|c|c|c|c|}
\hline \multirow[t]{2}{*}{ Epoch } & \multicolumn{9}{|c|}{ VERITAS Results } & \multicolumn{3}{|c|}{ Fermi LAT Results } \\
\hline & $\begin{array}{c}\text { Exposure } \\
\text { (hr) }\end{array}$ & $\begin{array}{c}\mathrm{ON}+\text { OFF Region }^{\mathrm{a}} \\
\text { Events }\end{array}$ & $\begin{array}{l}\text { Excess } \\
\text { Events }\end{array}$ & $\begin{array}{l}\text { Signal } \\
(\sigma)\end{array}$ & $\begin{array}{l}\text { Spectral Reconstruction } \\
\text { Range }(\mathrm{GeV})\end{array}$ & $\begin{array}{c}\text { Index } \\
\Gamma\end{array}$ & $\begin{array}{l}\text { Flux }>120 \mathrm{GeV} \\
\left(\times 10^{-7} \mathrm{~m}^{-2} \mathrm{~s}^{-1}\right)\end{array}$ & $\begin{array}{c}\text { Percent } \\
\text { Crab }(\%)\end{array}$ & $\chi^{2} / \mathrm{DOF}$ & $\begin{array}{c}\text { Index } \\
\alpha\end{array}$ & $\begin{array}{l}\text { Curvature } \\
\beta\left(\times 10^{-2}\right)\end{array}$ & $\begin{array}{c}\text { Flux }[0.1-300 \mathrm{GeV}] \\
\left(\times 10^{-8} \mathrm{~cm}^{-2} \mathrm{~s}^{-1}\right)\end{array}$ \\
\hline $2009^{b}$ & 28.5 & $3264 / 19635$ & 423 & 8.5 & $120-750$ & $3.8 \pm 0.3$ & $2.1 \pm 0.3$ & 4.6 & $3.2 / 6$ & $1.73 \pm 0.07$ & & $8.3 \pm 1.3$ \\
\hline $2013^{\mathrm{d}}$ & 66.8 & $12869 / 76307$ & 1675 & 14.4 & $100-750$ & $4.5 \pm 0.2$ & $1.02 \pm 0.08$ & 2.2 & $7.5 / 9$ & $1.77 \pm 0.09$ & $\cdots$ & $6.3 \pm 1.2$ \\
\hline Contemp. ${ }^{\mathrm{e}}$ & 109.9 & $20322 / 120734$ & 2638 & 18.1 & $100-750$ & $4.2 \pm 0.3$ & $1.30 \pm 0.08$ & 2.8 & $21.2 / 9$ & $1.77 \pm 0.05$ & $\ldots$ & $7.7 \pm 0.7$ \\
\hline Full $^{\mathrm{f}}$ & $\ldots$ & $\ldots$ & $\ldots$ & $\ldots$ & $\ldots$ & $\ldots$ & $\ldots$ & $\ldots$ & $\ldots$ & $1.64 \pm 0.06$ & $(2.7 \pm 0.8)$ & $7.37 \pm 0.04$ \\
\hline
\end{tabular}

Notes.

${ }^{a}$ Gamma-ray signal calculated according to Li \& Ma (1983), with the ratio between the ON and OFF region sizes of $\alpha=0.167,0.167$, and 0.200 in 2009, 2011, and 2013, respectively.

${ }^{\mathrm{b}}$ MJD 54881-54888, 54937-54943, 54968-54982, 54994-55003.

${ }^{c}$ MJD 55595-55604, 55620-55629, 55647-55662, 55677-55689, 55706-55711.

d MJD 56334-56341, 56358-56374, 56384-56400, 56413-56428, 56441-56447.

${ }^{e}$ Contemporaneous: includes all 2009, 2011, and 2013 epochs summarized above.

${ }^{\mathrm{f}}$ Fermi LAT data between MJD 54682 and 56452. Data fit with a log-parabolic model which includes absorption by the Gilmore et al. (2012) EBL model. 


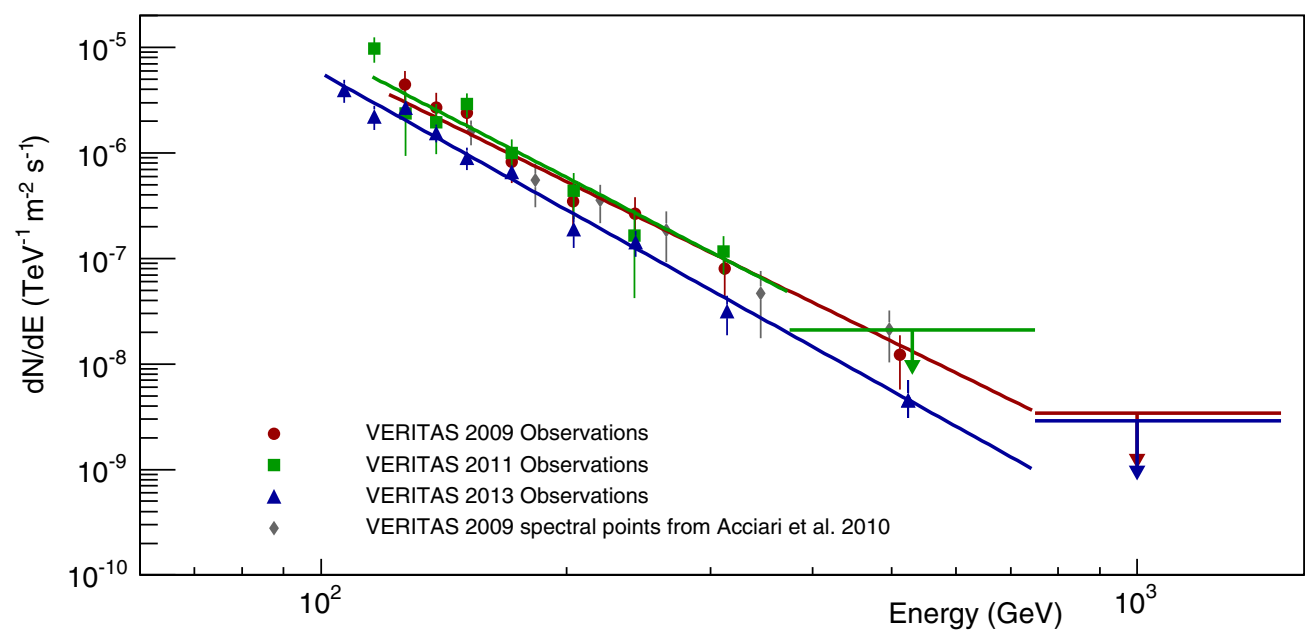

Figure 1. Observed VHE spectra of PKS 1424+240 derived from three years of VERITAS observations. The 2009, 2011, and 2013 spectral results are shown with $1 \sigma$ error bars. The spectrum from Acciari et al. (2010) is also shown in gray. See Table 1 for details.

(A color version of this figure is available in the online journal.)

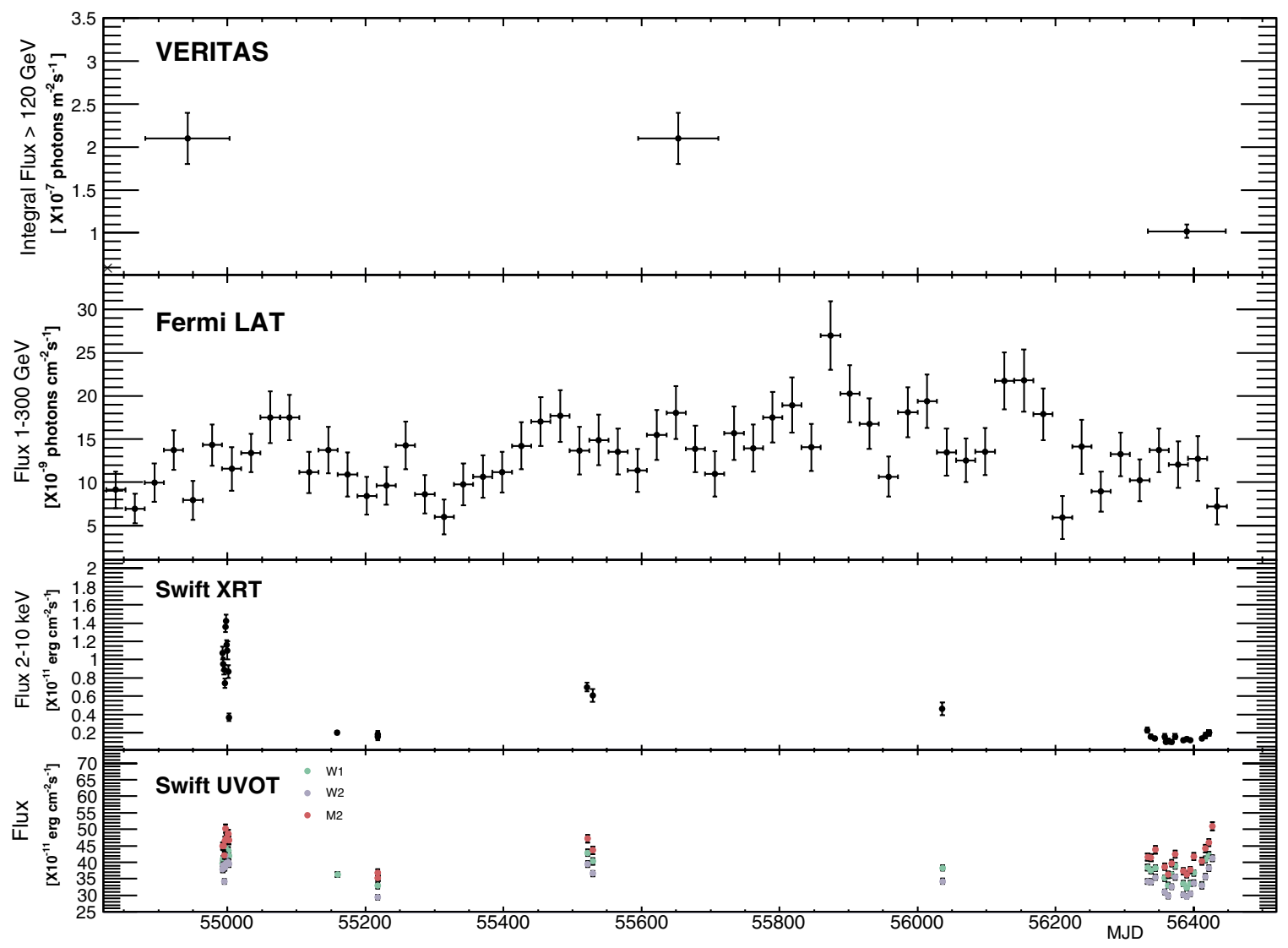

Figure 2. VERITAS, Fermi LAT, and Swift X-ray and UV light curves for PKS 1424+240.

(A color version of this figure is available in the online journal.)

isotropic component, which is the sum of the extragalactic diffuse gamma-ray emission and the residual charged particle background, are modeled using the recommended files. ${ }^{71}$ The flux systematic uncertainty is estimated to be approximately $5 \%$ at $560 \mathrm{MeV}$ and under $10 \%$ at $10 \mathrm{GeV}$ and above.

\footnotetext{
71 The files used were gal_2yearp7v6_v0.fits for the Galactic diffuse and iso_p7v6source.txt for the isotropic diffuse component available at http://fermi.gsfc.nasa.gov/ssc/data/access/lat/ BackgroundModels.html.
}

The data are fit with power-law models for each of three contemporaneous epochs in 2009, 2011, and 2013; they show no significant variations (see Table 1). The three epochs were also combined (referred to as "Contemp." in Table 1) and fit with a power law. Additionally, an extended LAT data set (MJD 54682 to 56452) is analyzed using the binned-likelihood method. The larger data set is fit with a curved log-parabolic model including EBL absorption with the Gilmore et al. (2012) EBL model, since there is a significant (TS > 9; Mattox et al. 1996) detection up to 


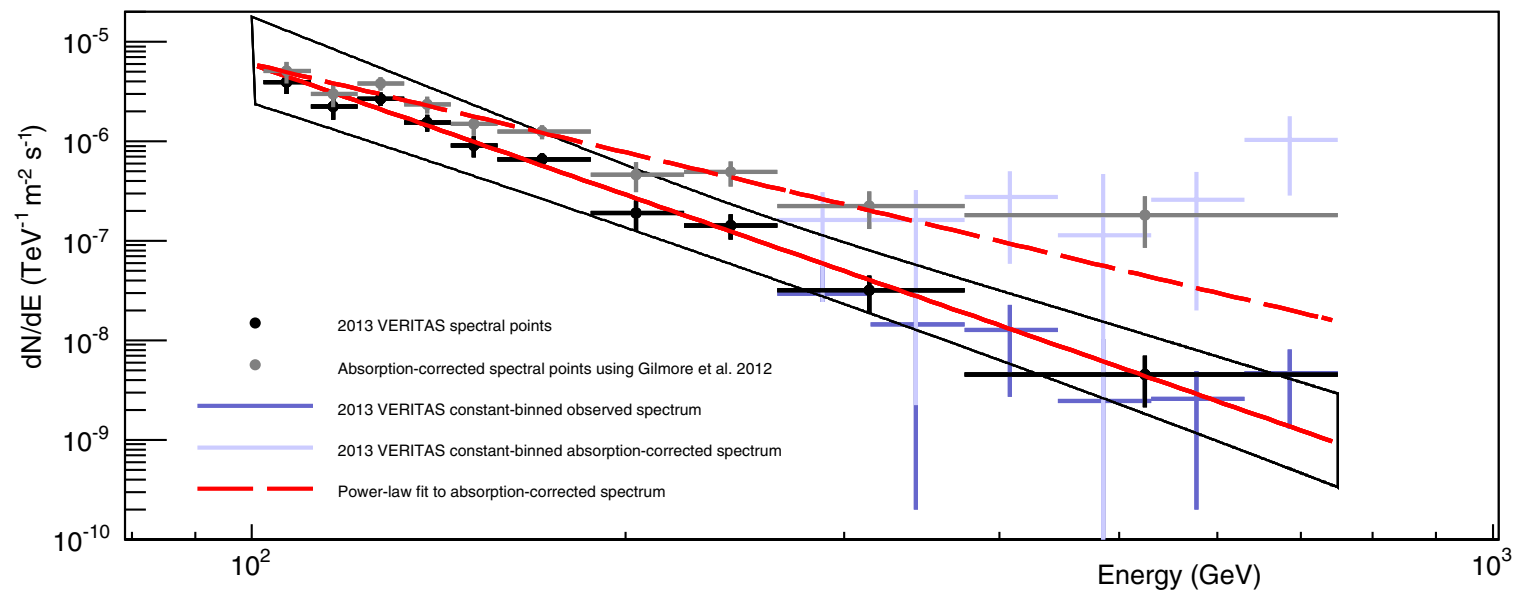

Figure 3. VHE spectrum derived from the 2013 VERITAS dataset, with significant detection between 100 and $750 \mathrm{GeV}$ (black points). The solid red line represents the power-law fit to the observed data. These data are shown with an envelope representing a $40 \%$ systematic error on the source flux and index error of \pm 0.3 . The data are also shown after correction for EBL-absorption by Gilmore et al. (2012), assuming $z=0.6035$ (gray points), along with the power-law (long-dashed line) fit to the absorption-corrected data. In blue, spectral points above $250 \mathrm{GeV}$ derived with constant binning are shown. None of the individual points above $400 \mathrm{GeV}$ is statistically significant in this representation.

(A color version of this figure is available in the online journal.)

$300 \mathrm{GeV}$. The contemporaneous 2009 and 2013 data are shown in Figure 4.

The data above $1 \mathrm{GeV}$ are also analyzed in 28 day bins (see Figure 2). This light curve displays variability, with a probability of $\sim 1 \times 10^{-11}$ of being steady $\left(\chi^{2}=159.7\right.$ with $57 \mathrm{DOF}$ ). However, a search for variability above $10 \mathrm{GeV}$ using the Bayesian Block method from Scargle et al. (2013) with $1 \%$ specified as the acceptable fraction of false positives shows no evidence of variability, in agreement with the lack of significant variability found above $10 \mathrm{GeV}$ in the Fermi LAT hard sources catalog (1FHL; Ackermann et al. 2013).

\subsection{Swift $X R T$}

The X-ray Telescope (XRT) on board the Swift satellite (Gehrels et al. 2004) is a focusing XRT sensitive to photons with energy between 0.2 and $10 \mathrm{keV}$. Thirty observations of PKS $1424+240$ summing to $51 \mathrm{ks}$ have been collected between 2009 June 11 and 2013 May 10 (MJD 54993 and 56422), inclusive. Observations were taken in photon counting mode with the count rate ranging from 0.1 to 1.1 counts per second. Pile-up effects are accounted for when the count rate exceeds 0.5 counts per second using an annular source region, with a 1-2 pixel inner radius and a 20 pixel outer radius. The data are analyzed as described in Burrows et al. (2005) with HEASOFT 6.9 and XSPEC version 12.6.0.

For spectral fitting, the photons are grouped by energy to require a minimum of 20 counts per bin, and fit with an absorbed power law (tbabs (po)) between 0.3 and $10 \mathrm{keV}$, fixing the neutral hydrogen column density to $3 \times 10^{20} \mathrm{~cm}^{-2}$, as quoted in Kalberla et al. (2005). The data are also fit with an absorbed log-parabolic model (tbabs(logpar)), finding curvature parameters consistent with zero. Due to the lack of curvature, we only discuss the power-law fitted parameters here.

The $2-10 \mathrm{keV}$ integral flux values are derived for each observation and shown in Figure 2. The X-ray light curve is clearly variable, with a constant fit giving a $\chi^{2}$ of 2200 for 30 DOF. X-ray energy spectra are extracted for the highest and lowest states (from MJD 54997 and 56368, respectively). The high and low flux states differ by a factor of $\sim 10$ and have photon indices of $\alpha=2.36 \pm 0.04$ and $\alpha=2.8 \pm 0.1$, respectively. These $\mathrm{X}$-ray states correspond to $2-10 \mathrm{keV}$ rest frame luminosities of at least $2.5 \times 10^{46} \mathrm{erg} \mathrm{s}^{-1}$ and $2.4 \times$ $10^{45} \mathrm{erg} \mathrm{s}^{-1}$, respectively, assuming $z=0.6035$. In order to represent the intrinsically emitted SED, the spectra corrected for the column density absorption are shown in Figure 4.

\subsection{Swift UVOT}

Swift-UVOT exposures were taken with UVW1, UVM2, and UVW2 filters (Poole et al. 2008). The UVOT photometry is performed using HEASoft uvotsource. The circular source region has a $5^{\prime \prime}$ radius and the background region consists of several circles of $15^{\prime \prime}$ radius of nearby empty sky. The results are reddening-corrected using the $E(B-V)$ coefficients in Schlegel et al. (1998). The Galactic extinction coefficients are applied according to Fitzpatrick (1999). The uncertainty in the reddening $E(B-V)$ is the largest source of error. The UV light curve is shown in Figure 2, with the UV flux values corresponding to the high and low X-ray states plotted in Figure 4. UV variability is apparent, with a pattern similar to the X-ray band.

\section{ABSORPTION-CORRECTED BROADBAND SED}

Two broadband SEDs of PKS 1424+240 are shown in Figure 4, corresponding to relatively high and low states. Two inset plots show the absorption-corrected VHE data according to the EBL model in Gilmore et al. (2012). There is an indication of spectral hardening at the highest energies in the absorption-corrected VHE spectrum. Similar results are seen when absorption correction is done according to a variety of EBL models, such as Domínguez et al. (2011), Finke et al. (2010), and Franceschini et al. (2008).

The contemporaneous LAT data are also shown in the insets of Figure 4, but the correction for EBL absorption is $<1 \%$ at the highest energy LAT spectral points. Spectral results derived from the full LAT observations are also shown and are consistent with the VERITAS observations. The synchrotron peaks are shown with Swift XRT and UVOT observations from relatively high and low states. Since the synchrotron peak is known to be above UV energies (e.g., Acciari et al. 2010), these observations constrain the location of the synchrotron peak $\left(10^{15}-10^{16} \mathrm{~Hz}\right)$ 


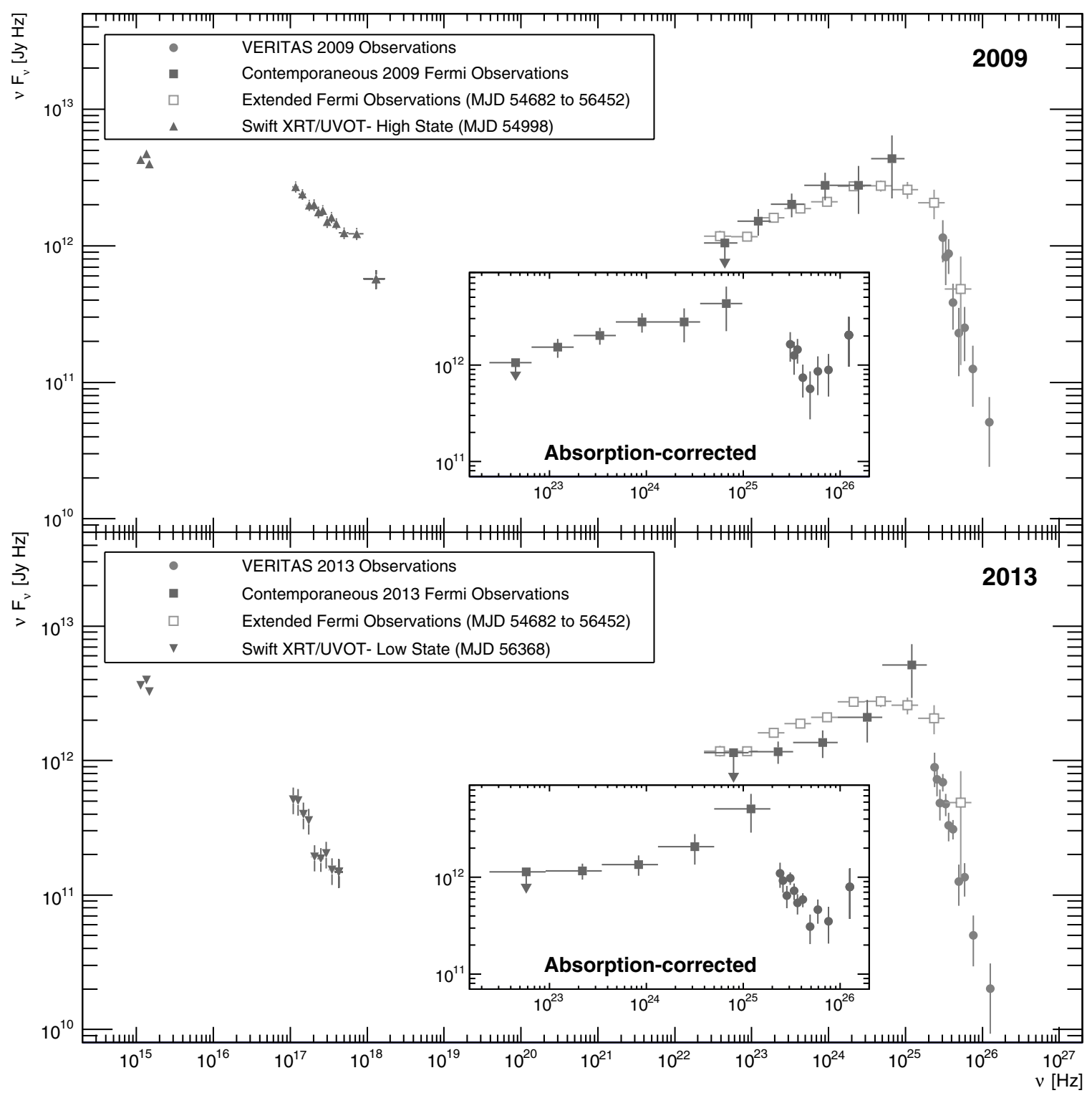

Figure 4. Two broadband SEDs of PKS 1424+240, corresponding to a relatively high (upper panel) and a low (lower panel) state. Within the inset, the VHE data are corrected for absorption using the Gilmore et al. (2012) EBL model for $z=0.6035$. The contemporaneous LAT data above $100 \mathrm{MeV}$ are shown along with the spectral results from full LAT observations. The Swift XRT and UVOT observations for relatively low and high states are also shown, after correction for absorption by the Milky Way column density.

during relatively low and high synchrotron flux states. The source is not detected between 14 and $195 \mathrm{keV}$ by the Burst Alert Telescope on board Swift in 70 months of data (Baumgartner et al. 2013).

\section{DISCUSSION}

The blazar PKS $1424+240$ resides at $z \geqslant 0.6035$, with a VHE flux that is significantly attenuated by the EBL. Discovery observations of this source by VERITAS have shown a marginal indication of spectral hardening at the highest energies, after correction by the EBL (Furniss et al. 2013). While a similar effect is seen in the deep observations obtained in 2013, the significance of the effect remains marginal because of the lower overall flux level during this epoch. In both epochs the data are consistent with a simple power law, even after correction for absorption by the EBL. If the indication of spectral hardening could be confirmed, one possible explanation would be an overestimation of the EBL density, although the results shown us one of the lowest density EBL models currently available, which approaches the galaxy count lower limits of the EBL density at $z \sim 0$.

The possible spectral hardening is of great interest, because if it is not from overestimation of the EBL, there are a number of physical mechanisms that can produce hardening with increasing energy. Second-order synchrotron self-Compton emission, pair-cascades initiated by pion decay in hadronic emission scenarios (Böttcher et al. 2013), or internal photon-photon absorption (Aharonian et al. 2008) can produce hard components at high energy. There are also scenarios that describe spectral hardening as arising from hadronic cosmic-ray line-of-sight interactions with the cosmic microwave background and EBL. These processes can produce secondary gamma-rays close to the observer, hardening the observed VHE spectrum (Essey \& Kusenko 2010a, 2010b, 2012; Essey et al. 2011; Murase et al. 2012; Razzaque et al. 2012; Prosekin et al. 2012; Aharonian et al. 2013; Zheng \& Kang 2013; Kalashev et al. 2013; Inoue et al. 2014). This component is expected to become dominant 
at high energies where the EBL opacity is greater than $\sim 2$ and is not expected to vary on timescales shorter than about a year. The VERITAS observations above $310 \mathrm{GeV}$ (where $\tau=2$ according to Gilmore et al. 2012 and Kneiske \& Dole 2010) do not show significant variability between 2009 and 2013, nor can they strongly exclude it. More exotic theories, involving Lorentz invariance violation (Urry \& Piran 2008) or axion-like particles, might also produce spectral hardening at high energies, e.g., Sánchez-Conde (2009).

The blazar can be categorized as a high-synchrotron-peaked (HSP) BL Lac, with a synchrotron peak above $10^{15} \mathrm{~Hz}$ (Abdo et al. 2010) and an isotropic luminosity above $400 \mathrm{GeV}$ of $1.03 \times 10^{44} \mathrm{erg} \mathrm{s}^{-1}$. At $z \geqslant 0.6035$, it is apparent that PKS 1424+240 represents a powerful tool for studying the intrinsic emission mechanism(s) within blazar jets, extragalactic cosmic-ray propagation, and the propagation of VHE photons across extragalactic space. Future studies will benefit from additional VHE observations as well as from any additional information that will be obtained about the redshift, e.g., from HST/STIS UV observations.

This research is supported by grants from the U.S. Department of Energy Office of Science, the U.S. National Science Foundation, and the Smithsonian Institution; by NSERC in Canada; by Science Foundation Ireland (SFI 10/RFP/AST2748); and by STFC in the UK. We acknowledge the excellent work of the technical support staff at the Fred Lawrence Whipple Observatory and at the collaborating institutions in the construction and operation of the instrument.

Support for program HST-GO-12863 was provided by NASA, awarded through the Space Telescope Science Institute, operated by the Association of Universities for Research in Astronomy, Inc., for NASA, under contract NAS 5-26555.

The Fermi LAT Collaboration acknowledges support from a number of agencies and institutes for both development and the operation of the LAT as well as scientific data analysis. These include NASA and DOE in the United States; CEA/Irfu and IN2P3/CNRS in France; ASI and INFN in Italy; MEXT, KEK, and JAXA in Japan; and the K. A. Wallenberg Foundation, the Swedish Research Council, and the National Space Board in Sweden. Additional support from INAF in Italy and CNES in France for science analysis during the operations phase is also gratefully acknowledged.

Facilities: VERITAS, Fermi, Swift

Note Added in Proof. Subsequent to the submission of this paper, the MAGIC collaboration reported results on observations of PKS 1424+240 in 2009-2011 (Aleksic et al. 2014). Their new results do not change the conclusions of this paper.

\section{REFERENCES}

Abdo, A. A., Ackermann, M., Agudo, I., et al. 2009, ApJS, 183, 46 Abdo, A. A., Ackermann, M., Agudo, I., et al. 2010, ApJ, 716, 30 Abramowski, A., Acero, F., Aharonian, F., et al. 2013, A\&A, 550, 4 Acciari, V. A., Aliu, E., Arlen, T., et al. 2010, ApJL, 708, L100 Ackermann, M., Ajello, M., Allafort, A., et al. 2012, Sci, 338, 1190 Ackermann, M., Ajello, M., Allafort, A., et al. 2013, ApJS, 209, 34 Aharonian, F., Akhperjanian, A. G., Bazer-Bachi, A. R., et al. 2006, Natur, 440,1018

Aharonian, F., Essey, W., Kusenko, A., \& Prosekin, A. 2013, PhRvD, 87, 063002

Aharonian, F., Khangulyan, D., \& Costamante, L. 2008, MNRAS, 387, 1206

Albert, J., Aliu, E., Anderhub, H., et al. 2008, Sci, 320, 1752

Aleksic, J., Ansoldi, S., Antonelli, L. A., et al. 2014, A\&A, submitted (arXiv:1401.0464)

Atwood, W., Abdo, A., Ackermann, M., et al. 2009, ApJ, 697, 1071

Baumgartner, W. H., Tueller, J., Markwardt, C. B., et al. 2013, ApJS, 207, 19

Böttcher, M., Reimer, A., Sweeney, K., \& Prakash, A. 2013, ApJ, 768, 54

Burrows, D. N., Hill, J. E., Nousek, J. A., et al. 2005, SSRv, 120, 165

Cogan, P. 2008, in Proc. 30th Int. Cosmic Ray Conf., Vol. 3, The VERITAS Gamma-ray Analysis Suite, ed. R. Caballero et al. (Mexico City, Mexico: Universidad Nacional Autónoma de México), 1385

Domínguez, A., Primack, J., Rosario, D. J., et al. 2011, MNRAS, 410, 2556

Essey, W., Kalashev, O., Kusenko, A., \& Beacom, J. 2011, APJ, 731, 51

Essey, W., \& Kusenko, A. 2012, ApJL, 751, L11

Essey, W., \& Kusenko, A. 2010a, APh, 33, 81

Essey, W., \& Kusenko, A. 2010b, PhRvL, 104, 141102

Finke, J., Razzaque, S., \& Dermer, C. 2010, ApJ, 712, 238

Fitzpatrick, E. 1999, PASP, 111, 63

Fomin, V. P., Sepanian, A. A., Lamb, R., et al. 1994, APh, 2, 137

Franceschini, A., Rodighiero, G., \& Vaccari, M. 2008, A\&A, 487, 837

Furniss, A., Williams, D. A., Danforth, C., et al. 2013, ApJL, 768, L31

Gehrels, N., Chincarini, G., Giommi, P., et al. 2004, ApJ, 611, 1005

Gilmore, R., Somerville, R., Primack, J., \& Dominguez, A. 2012, MNRAS, 422, 3189

Holder, J., Atkins, R. W., Badran, H. M., et al. 2006, APh, 25, 391

Horns, D., \& Meyer, M. 2012, JCAP, 2, 33

Inoue, Y., Kalashev, O. E., \& Kusenko, A. 2014, APh, 54, 118

Kalashev, O. E., Kusenko, A., \& Essey, W. 2013, PhRvL, 111, 041103

Kalberla, P., Burton, W., Hartmann, D., et al. 2005, A\&A, 440, 775

Kneiske, T. M., \& Dole, H. 2010, A\&A, 515, 19

Li, T., \& Ma, Y. 1983, ApJ, 272, 317

Murase, K., Dermer, C., Takami, H., \& Migliori, G. 2012, ApJ, 749, 63

Mattox, J., Bertsch, D., Chiang, J., et al. 1996, ApJ, 461, 396

Nikishov, A. I. 1962, JETP, 14, 393

Nolan, P. L., Abdo, A. A., Ackermann, M., et al. 2012, ApJS, 199, 31

Poole, T., Breeveld, A., Page, M. J., et al. 2008, MNRAS, 383, 627

Prokoph, H. 2013, PhD thesis, Humboldt Univ., Berlin

Prosekin, A., Essey, W., Kusenko, A., \& Aharonian, F. 2012, ApJ, 757, 183

Razzaque, S., Dermer, C., \& Finke, J. D. 2012, ApJ, 745, 196

Rolke, W. A., López, A. M., \& Conrad, J. 2005, NIMPA, 551, 493

Sánchez-Conde, M., Paneque, D., Bloom, E., Prada, F., \& Domínguez, A. 2009, PhRvD, 79, 123511

Scargle, J., Norris, J., Jackson, B., \& Chiang, J. 2013, ApJ, 764, 167

Shlegel, D., Finkbeiner, D., \& Davis, M. 1998, ApJS, 500, 525

Urry, J., \& Piran, T. 2008, PhRvD, 78, 124010

Werner, M., Roellig, T. L., Low, F. J., et al. 2004, ApJS, 154, 1

Zheng, Y. G., \& Kang, T. 2013, ApJ, 764, 113 